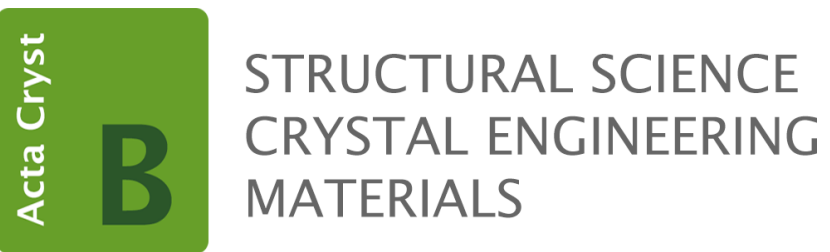

Volume 77 (2021)

Supporting information for article:

Size-dependent strain in fivefold twins of gold

Hao Wu, Rong Yu, Jing Zhu, Wei Chen, Yadong Li and Tao Wang 


\section{Structural models}

A typical decahedron in a diameter of about $7 \mathrm{~nm}$ was built using homogeneous strain model ${ }^{(B a g l e y, ~ 1965)}$, and the atomic positions in this particle are given as follow,

$$
\begin{gathered}
l \cdot\left[\begin{array}{c}
0 \\
0 \\
x_{i, j, k, l} \\
y_{i, j, k, l} \\
z_{i, j, k, l}
\end{array}\right]=\left\{\begin{array}{c}
i=1, j=0, k=0 \\
3 \leq l \leq 21
\end{array}\right] j \cdot A^{i-1} \cdot\left[\begin{array}{c}
0.2454 \\
0 \\
0.3032 / 2
\end{array}\right]+k \cdot A^{i} \cdot\left[\begin{array}{c}
0.2454 \\
0 \\
0.3032 / 2
\end{array}\right]+l \cdot\left[\begin{array}{c}
0 \\
0 \\
0.2454
\end{array}\right] \begin{array}{l}
1 \leq i \leq 5,0 \leq j \leq 13, \\
1 \leq k \leq 13, l \geq 0 \\
j+k \leq 16, j+k+l \leq 24 \\
3 \leq j / 2+k / 2+l \leq 21
\end{array} \\
A=\left[\begin{array}{ccc}
\cos (2 \pi / 5) & \sin (2 \pi / 5) & 0 \\
-\sin (2 \pi / 5) & \cos (2 \pi / 5) & 0 \\
0 & 0 & 1
\end{array}\right]
\end{gathered}
$$

where $i$ is the section number from 1 to $5, j, k$ and $l$ are indexes in each section, $A$ is the rotation matrix, $0.2454 \mathrm{~nm}$ and $0.3032 \mathrm{~nm}$ are parameters calculated in the homogeneous strain model ${ }^{(B a g l e y, 1965)}$ and the units for these coordinates are nanometers. Atomic projection positions on the XY plane and atomic positions on one of the twin boundaries in this particle are shown in Figure S1a and S1b respectively.
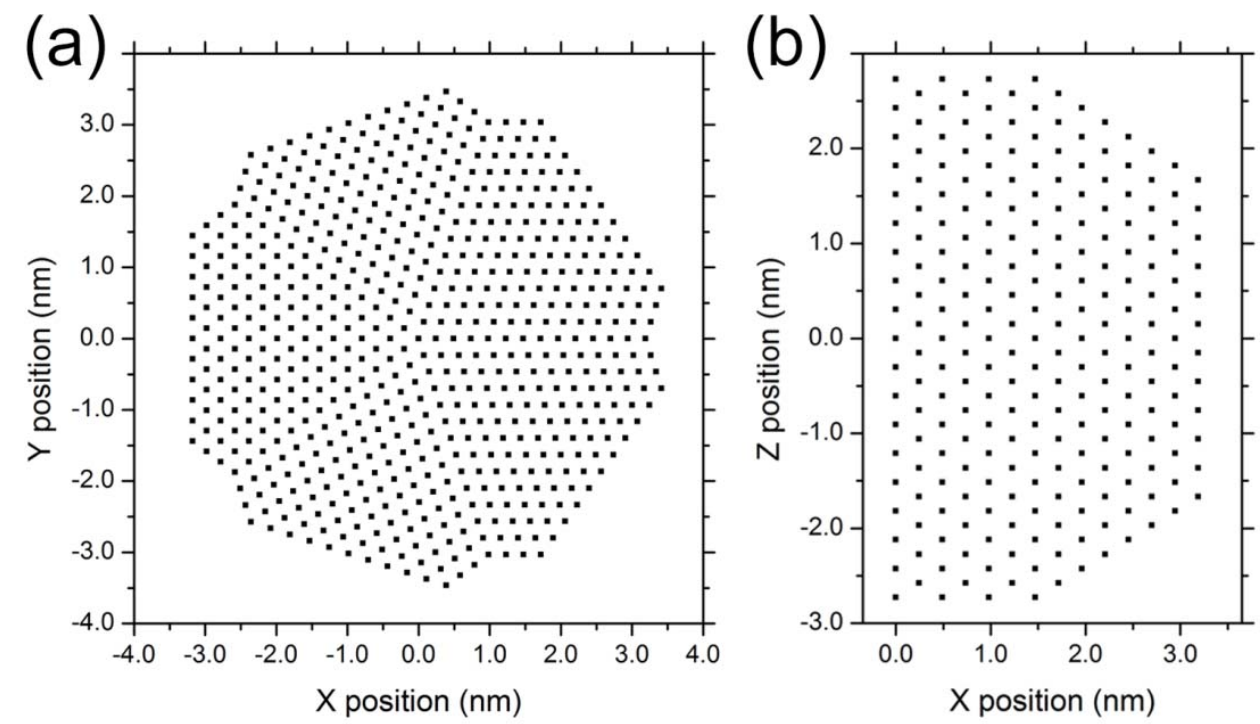
Figure S1. Atomic projection positions on XY plane (a) and atomic positions on one of the twin boundaries (b) in a typical Marks decahedron built using homogeneous strain model.

\section{Effects of residual aberrations and sample tilt}

Residual aberrations (2-fold astigmatism, coma and so on), tilt of the samples away from the accurate axis, and vibrations are inevitable in high resolution TEM observations. The inaccuracy of further quantitative measurement depends on these facts and sometimes cannot be neglected. High resolution TEM image simulation is necessary to estimate the inaccuracy caused by the imperfect imaging condition.

Different imaging conditions are used in imaging simulation including vibration, 2-fold astigmatism, 3-fold astigmatism, coma, and sample tilt. Imaging conditions are shown in Table S1. The astigmatism, coma or sample tilt, when included, was set to be in the x direction. Quantitative measurements of positions of atomic columns were performed in each image and the average displacements between the positions of atomic columns got from the simulation images and the projected atomic positions were calculated as shown in Table S1. As discussed below, the atomic displacements from the imaginary flat planes in a DP in size of about $7 \mathrm{~nm}$ are no more than $24 \mathrm{pm}$. It means that we'd better make the inaccuracy of the quantitative measurements induced by the imperfect of the imaging condition as small as possible. Increase of the average displacements due to the residual aberrations (2-fold astigmatism, 3-fold astigmatism and coma) and vibration is very small. However tilt of the sample is deadly for the quantitative measurements especially for tilt more than $8 \mathrm{mrad}$. As residual aberrations can be measured during the experiments, the vibrations of sample tilt have to be estimated through image simulations.

Table S1. Quantitative measurements in images under different imaging conditions.

\begin{tabular}{|c|c|c|}
\hline Index & Image conditions & Average displacements (pm) \\
\hline$(1)$ & perfect & 0.8 \\
\hline$(2)$ & vibration $(20 \mathrm{pm})$ & 1.0 \\
\hline$(3)$ & vibration $(20 \mathrm{pm})+2$-fold astigmatism $(2 \mathrm{~nm})$ & 1.0 \\
\hline
\end{tabular}




\begin{tabular}{|c|c|c|}
\hline$(4)$ & vibration $(20 \mathrm{pm})+3$-fold astigmatism $(20 \mathrm{~nm})$ & 0.9 \\
\hline$(5)$ & vibration $(20 \mathrm{pm})+$ coma $(20 \mathrm{~nm})$ & 1.1 \\
\hline$(6)$ & vibration $(20 \mathrm{pm})+$ tilt $(4 \mathrm{mrad})$ & 1.4 \\
\hline$(7)$ & vibration $(20 \mathrm{pm})+$ tilt $(8 \mathrm{mrad})$ & 2.5 \\
\hline$(8)$ & vibration $(20 \mathrm{pm})+\operatorname{tilt}(12 \mathrm{mrad})$ & \\
\hline
\end{tabular}

Typical Cs-corrected HRTEM images of gold DPs in good or bad imaging conditions are shown in Figure S2a and S2b respectively, with the typical atomic columns enlarged shown in the corresponding insets. In Figure S2a the particle shows a much lower contrast than the other one (due to the channeling effect), and the peaks are very round with homogeneous aureole around. Tilt of the samples in Figure S2a and $\mathrm{b}$ are about $5 \mathrm{mrad}$ and $12 \mathrm{mrad}$ respectively.

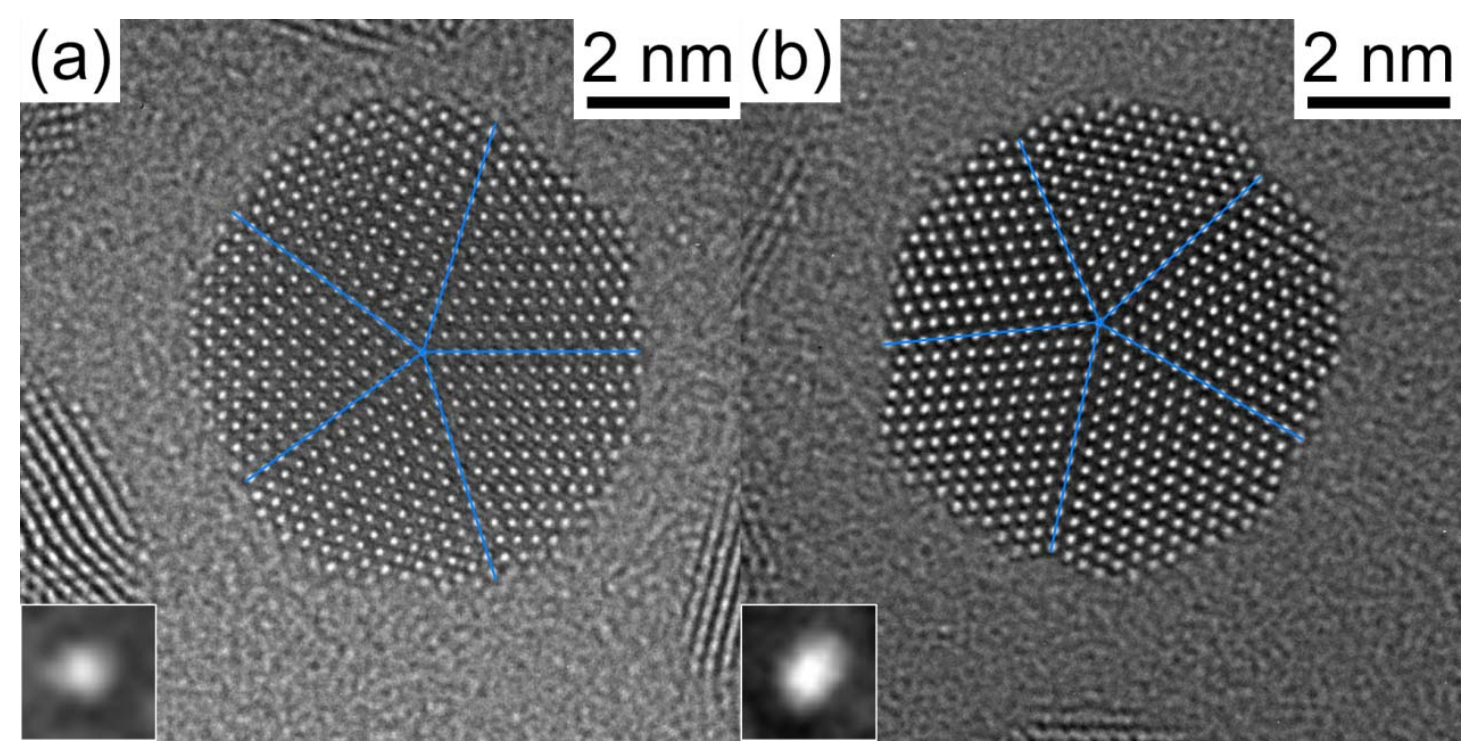

Figure S2. Typical Cs-corrected HRTEM images of gold DPs, suitable to quantitative measurement (a) and not suitable to quantitative measurement (b). Images of a typical atomic column are enlarged and shown in the corresponding insets.

\section{Strain models}

Since the strain along the fivefold axis in decahedra is indistinctive, researchers usually focus on the strain distribution in the plane normal to the fivefold axis. Here 
we discuss the shape of (002) planes in several structure models, such as

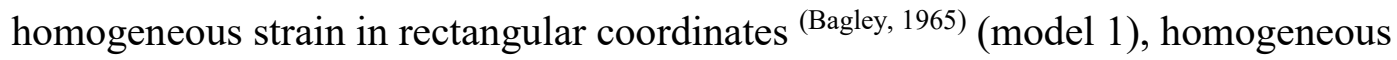
strain in cylindrical coordinates ${ }^{(H o w i e ~ \& ~ M a r k s, ~ 1984) ~(m o d e l ~ 2) ~ a n d ~ t h e ~ d i s c l i n a t i o n ~ m o d e l ~}$ (de Wit, 1972) (model 3). The strain distributions in these models are shown in formulae (3)-(5), respectively,

$$
\begin{gathered}
{\left[\begin{array}{l}
\varepsilon_{x} \\
\varepsilon_{y}
\end{array}\right]=\left[\begin{array}{c}
-\frac{1}{2} \varepsilon_{D} \\
\frac{1}{2} \varepsilon_{D}
\end{array}\right], \quad \varepsilon_{D}=\sqrt{2} \cdot \tan (\pi / 5)-1} \\
{\left[\begin{array}{c}
\varepsilon_{\rho} \\
\varepsilon_{\theta}
\end{array}\right]=\left[\begin{array}{c}
0 \\
\varepsilon^{\prime}{ }_{D}
\end{array}\right], \quad \varepsilon_{D}^{\prime}=(\pi / 5) / \arctan (\sqrt{2} / 2)-1} \\
{\left[\begin{array}{c}
\varepsilon_{\rho} \\
\varepsilon_{\theta}
\end{array}\right]=\left[\begin{array}{c}
\left.\frac{\Omega}{4 \pi}\left[\frac{1-2 v}{1-v} \ln \left(\frac{\rho}{R}\right)-\frac{v}{1-v}\right]\right], \quad \Omega=2 \pi-10 \arctan (\sqrt{2} / 2) \\
\frac{\Omega}{4 \pi}\left[\frac{1-2 v}{1-v} \ln \left(\frac{\rho}{R}\right)+1\right]
\end{array}\right],}
\end{gathered}
$$

Where $x, y$ are coordinates in rectangular coordinates, $\rho, \theta$ are coordinates in cylindrical coordinates, $R$ is the radius of this fivefold structure induced in disclination model. The strain distributions in model 2 and 3 are not homogeneous in rectangular coordinates, so they are usually considered as inhomogeneous models.

According to the strain distribution described above, the shapes of (002) planes in initial FCC structure and these models are calculated and shown in Figure S3a. Clearly, the (002) plane in model 1 remains flat, while those in model 2 and 3 bend. Quadratic equations were used to fit the bending of the (002) planes as shown in Figure S3a, and they match well with each other. It means that in the inhomogeneous models, the inhomogeneous part of the strain distribution mainly comes from the bending of the (002) planes and the shape of the bending can be well fitted with quadratic equations. In model $3, v=0.42$ for gold $^{(\text {Levy et al., 2001) }}$ and $R=R_{0}=\sqrt{3 / 2}$ are used in the calculations in Figure S3a. As a result of $v$ being very close to 0.5 , the shape of (002) planes changes with $\mathrm{R}$ very slightly. $R=R_{0}$ and $R=10 R_{0}$ were used in calculating the shape of (002) planes in model 3. As shown in Figure S3b, the shape 
changes only slightly even $R$ changes 10 times. The difference in the bending parameter $p$ is so small that it is assumed to be the same for all (002) planes in particles smaller than $10 \mathrm{~nm}$. The angular increase due to the bending of crystal planes is schematically (exaggerated) shown in Figure S3c. Quantitatively, $\Delta \theta=4|p| / 9$ in radian.
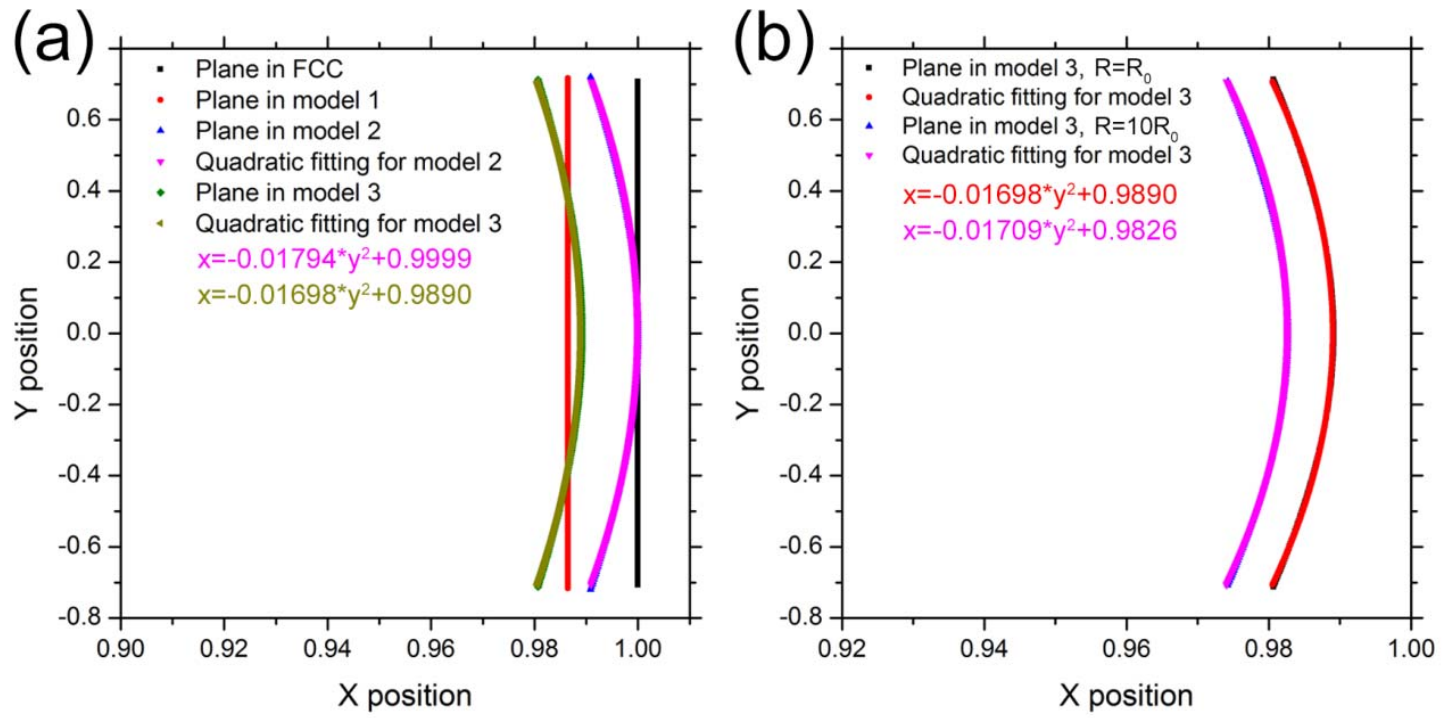

(c)

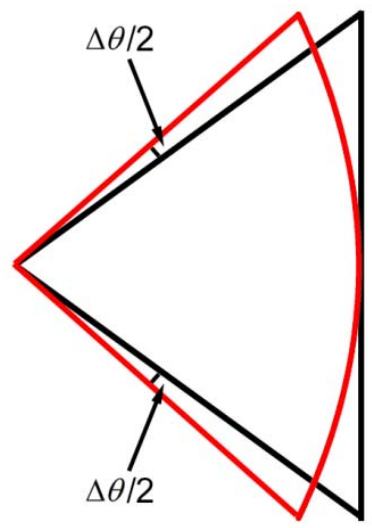

Figure S3. (a) Shapes of the (002) planes in FCC structure and several models, with quadratic fitting used for the bending of crystal planes in the inhomogeneous models. (b) Shapes of the (002) planes in model 3 with different parameters. (c) Schematics showing the angular increase due to the bending of lattice planes. 


\section{References}

Bagley, B. G. (1965). Nature 208, 674-675.

de Wit, R. (1972). Journal of Physics C: Solid State Physics 5, 529-534.

Howie, A. \& Marks, L. D. (1984). Philosophical Magazine A 49, 95-109.

Levy, M., Bass, H. E., Stern, R. R., Keppens, V. \& Furr, L. (2001). Handbook of Elastic Properties of Solids, Liquids, and Gases: Elastic Properties of Solids: Theory, Elements and Compounds, Novel materials, Technological Materials, Alloys, and Building materials. San Diego: Academic Press. 